Rev. Adm. Saúde (On-line), São Paulo, v. 19, n.77: e189, out. - dez. 2019, Epub 25 nov. 2019 http://dx.doi.org/10.23973/ras.77.189

ARTIGO ORIGINAL

\title{
Perfil epidemiológico das lesões por pressão em um hospital escola no Oeste do Paraná
}

Epidemiological profile of pressure injuries in a teaching hospital in Paraná West Region

\author{
Josni Tauffer ${ }^{1}$, Débora Cristina Ignásio Alves ${ }^{2}$, Bruna Tais Zack ${ }^{1}$, Manoela \\ Cristina Berticelli1, Maria Julia Navarro Kássim¹, Sabrina Kássia \\ Menegusso Carmello ${ }^{1}$
}

1. Enfermeiro(a). Residente em vigilância em saúde e controle de infecções no Hospital Universitário do Oeste do Paraná (HUOP) da Universidade Estadual do Oeste do Paraná (UNIOESTE)

2. Enfermeira, doutora em ciências da saúde. Professora adjunta de enfermagem da UNIOESTE

\section{RESUMO}

Objetivo: Descrever o perfil epidemiológico das lesões por pressão em um hospital escola de alta complexidade. Métodos: O presente estudo foi desenvolvido no Setor de Controle de Infecção do Hospital Universitário do Oeste do Paraná, pelo programa de Residência em Vigilância em Saúde e Controle de Infecção, caracterizado por estudo transversal, quantitativo, o qual ocorreu entre maio de 2016 a maio de 2018 através de tabelas de controle das lesões por pressão, relatórios em prontuário eletrônico e fichas de avalição pela comissão de curativos. Resultados: A amostra foi de 227 fichas de notificações de lesões por pressão, 161 (71\%) eram do sexo masculino, 195 $(85,9 \%)$ eram pacientes da cor branca. Com relação a idade, variou-se entre 23 dias a 93 anos com média de 54,8 anos (DP $\pm 21,6$ ) entre ambos os sexos. A média de dias de internação foi de 45 dias (DP \pm 42 ), e o tempo médio de surgimento da lesão foi de 18 dias (DP \pm 21 ) após a admissão. Um total de 206 pacientes (91\%) possuía algum tipo de comorbidade. Entre as clínicas/diagnóstico observou-se que houve predominância entre trauma, 
patologias neurológicas e patologias pulmonares, com 27,30\%, 26,00\% e $23,80 \%$ respectivamente. A variação de número de lesões por paciente foi de 1 a 5 lesões com média de 1,19. Dessas lesões cerca de 47,14\% eram em região sacral e $32,60 \%$ de todas as lesões eram descritas como estágio 1. Em relação ao desfecho dos casos 89 pacientes (39\%) evoluíram a óbito.

Conclusão: O conhecimento da realidade institucional proporciona ao profissional base cientifica para tomada de decisões. As práticas baseadas em evidência vêm cada vez mais tomando os espaços de prestação de assistência, contribuindo para aprimoramento das técnicas aos quais refletem diretamente no bom prognóstico dos pacientes aos quais são aplicados.

Palavras-chave: Lesão por Pressão; Epidemiologia; Enfermagem

\section{ABSTRACT}

Objective: To describe the epidemiological profile of pressure injuries in a highcomplexity teaching hospital. Methods: The present study was developed in the Infection Control Sector of the University Hospital of Western Paraná by the Residency Program in Health Surveillance and Infection Control, characterized by a cross-sectional quantitative study, which took place from May 2016 to May 2018 through pressure injury control tables, electronic medical record reports and assessment sheets by the dressing committee. Results: The sample consisted of 227 reports of pressure injury reports, 161 (71\%) were male, 195 (85.9\%) were white. Regarding age, it ranged from 23 days to 93 years with an average of 54.8 years $(S D \pm 21.6)$ between both sexes. The average hospital stay is 45 days ( $S D \pm 42$ ), and the average time of onset of the lesion was 18 days (SD \pm 21$)$ after admission. A total of 206 patients (91\%) had some kind of comorbidity. Among the clinics / diagnosis it was observed that there was a predominance between trauma, neurological and pulmonary pathologies, with $27.30 \%, 26.00 \%$ and $23.80 \%$ respectively. The number of lesions per patient ranged from 1 to 5 lesions with a mean of 1.19. About $47.14 \%$ of these lesions were in the sacral region and 32.60\% of all lesions were described as stage 1. Regarding the outcome of the cases, 89 patients (39\%) died. Conclusion: Knowledge of the institutional reality provides the professional with scientific basis for decision making. Evidence-based practices are increasingly taking over care spaces, contributing to the improvement of techniques that directly reflect the good prognosis of the patients to whom they are applied.

Keywords: Pressure Injury; Epidemiology; Nursing

\section{INTRODUÇÃO}


De acordo com a National Pressure Ulcer Advisory Panel (NPUAP), a terminologia de úlcera de pressão sofreu alteração para lesão por pressão (LP) e houveram alterações em sua descrição quanto ao seus estágios, o qual passou de números romanos para números arábicos. ${ }^{1}$ São consideradas um problema de saúde persistente, ocorrendo em diversos contextos da assistência, principalmente no ambiente hospitalar, comprometendo a segurança e a qualidade de vida do paciente, acarretando consequências devido ao sofrimento interposto e o maior risco de complicações resultando em aumento do tempo de internação e em elevados custos para a instituição hospitalar e para a família do paciente. ${ }^{2}$

Conforme a NPUAP, as LP podem causar danos à pele e/ou tecidos moles subjacentes. Formando-se geralmente sobre uma proeminência óssea ou relacionada com um dispositivo devido a uma intensa pressão prolongada e/ou de pressão em combinação com cisalhamento, a qual pode apresentar pele intacta ou uma úlcera aberta e pode ser doloroso. A tolerância do tecido mole para a pressão e cisalhamento também podem ser afetados pelo microclima, nutrição, perfusão, comorbidades e condição do tecido mole. ${ }^{1}$

O indivíduo acometido por LP tem um impacto negativo em sua qualidade de vida por desenvolver traumas físicos, além de psicológicos como, medo, ansiedade, isolamento entre outros. Em grande parte, por se tratar de agravo principalmente físico, a mortalidade de pacientes com LP demonstra-se expressiva sem que o prognóstico do acometido propicia o surgimento dessas lesões, como por exemplo pacientes debilitados nutricionalmente, com presença de comorbidades ou por causas extrínsecas como trauma. ${ }^{3}$

Estudos epidemiológicos relatam em média $10 \%$ a $69 \%$ de incidência em pacientes críticos, em diversos setores chega a 16,9\%.4,5,6 Em um estudo realizado em um hospital de ensino na China, a incidência de surgimento de LP em pacientes internados é de 0,63\%, o mesmo estudo compara dados europeus, os quais apresentam-se com incidência de 13,27\%.7 De acordo com a NPUAP a prevalência de úlcera de pressão nos hospitais dos Estados Unidos varia de $3 \%$ a $14 \%{ }^{1}$

Referente a temática evidenciou-se a necessidade de analisar o perfil epidemiológico da incidência de LP na instituição de inquérito. Este estudo tem como principal intuito contribuir com dados e informações sobre a ocorrência de LP, a fim de subsidiar outras pesquisas, contribuindo em evidência para prática assistencial no que se diz respeito a melhoria da qualidade prestada.

\section{MÉTODOS}

O presente estudo foi desenvolvido junto ao Setor de Controle de Infecção (SCIH) de hospital universitário público do interior do Paraná. Trata-se de um estudo transversal com abordagem quantitativa. Esta pesquisa teve a aprovação do Comitê de Ética em Pesquisa (CEP) sob o parecer no 30622301/2018, preservando a identidade dos envolvidos sem exposição de quaisquer dados que possibilitem sua identificação. 
A tabulação dos dados ocorreu entre maio de 2016 a maio de 2018 por meio de tabelas de controle das lesões por pressão. Esta coleta ocorreu através da busca passiva em prontuários eletrônicos, através da descrição em evolução diária em complementação da busca ativa setorial afim de contribuir para os dados pertinentes as características das lesões, e através de auxilio as fichas de lesão por pressão desenvolvidas através da Comissão de Curativos a qual tem por finalidade avaliar e descrever o acometimento de lesões em pacientes internados. Estes dados são informados através de tabelas próprias do $\mathrm{SCIH}$ da instituição onde constam variáveis do perfil sociodemográfico dos pacientes que vieram a desenvolver lesões por pressão.

As variáveis estudadas foram: sexo, idade, cor da pele, setor de internamento/clínica de diagnóstico, dias de internamento, tempo de desenvolvimento da lesão por pressão, comorbidades e quantidades de lesões. O teste de Friedman foi aplicado nas variáveis entre idade/tempo de internação e idade/tempo de desenvolvimento da lesão por pressão.

O teste qui-quadrado foi aplicado nas variáveis sexo/desfecho. As demais variáveis sofreram os testes de média.

No presente estudo, utilizou-se da aplicação dos testes de Friedman e quiquadrado para apresentação de valores médios afim de verificar a significância entre as variáveis. As apresentações dos resultados deram-se por números absolutos e porcentagens afim do proporcionar maior entendimento.

\section{RESULTADOS E DISCUSSÃO}

A amostra foi de 227 fichas de notificações de lesões sendo que 161 pacientes $(71 \%)$ eram do sexo masculino e $66(29 \%)$ do sexo feminino. Com relação à cor da pele, 195 pacientes (85,90\%) eram brancos, 19 (8,37\%) pardos, 3 $(1,31 \%)$ amarelos, $1(0,44 \%)$ negro e em 9 fichas, este dado não estava descrito.

De acordo com as notificações, a maioria dos pacientes tinha uma comorbidade. Estima-se que anualmente nos Estados Unidos entre um e três milhões de pessoas desenvolvem algum tipo de lesão por pressão. Entre eles, 60 mil morrem em decorrência de complicações secundárias. ${ }^{8}$ No Brasil, existe uma grande dificuldade em mensurar esses dados, porém alguns estudos relataram uma variação de 5,9\% a 94\%, na prevalência de lesões por pressão, apontando um custo médio de $\mathrm{R} \$ 33.000,00$, por paciente ao ano. ${ }^{9}$

A idade dos pacientes variou entre 23 dias a 93 anos (Tabela 1).

Tabela 1. Distribuição da amostra, de acordo com a faixa etária dos pacientes notificados.

\begin{tabular}{|l|r|r|}
\hline Faixa etária (anos) & $\mathbf{N}$ & $\%$ \\
\hline
\end{tabular}




\begin{tabular}{|l|r|r|}
\hline $0-20$ & 26 & 11,45 \\
\hline $21-40$ & 44 & 19,38 \\
\hline $41-60$ & 42 & 18,50 \\
\hline $61-80$ & 89 & 39,20 \\
\hline$>80$ & 26 & 11,46 \\
\hline Total & $\mathbf{2 2 7}$ & $\mathbf{1 0 0 , 0 0}$ \\
\hline
\end{tabular}

A idade média entre os pacientes foi de 54,8 anos (DP $\pm 21,6$ ), sendo entre os pacientes do sexo feminino a idade média de 56,7 anos (DP $\pm 20,4$ ) e entre os de sexo masculino 54,2 anos (DP $\pm 22,1$ ). Quando comparadas a idade, é notório perceber que o perfil muda de acordo com a especificidade do hospital. Foram encontrados dados diferentes dos demais achados na literatura, em que a faixa etária referida no trabalho é um dado isolado, porém, em hospital de referência da Amazônia é predominante a idade de 60 a 80 anos. Em dois estudos com características similares a média de idade era de 70 a 75 anos, já no Instituto de Saúde em Fortaleza, Brasil, 31\% dos pacientes estavam entre 18 a 25 anos. ${ }^{5-10,11,12}$

Os pacientes permaneceram em média 45 dias internados (DP \pm 42 ). Segundo o sexo, pacientes do sexo masculino ficaram, em média, 49 (DP \pm 44 ) dias internados e pacientes do sexo feminino, 34 ( $\mathrm{DP} \pm 33$ ) dias internadas. $\mathrm{O}$ tempo de desenvolvimento das lesões foi em média de 18 dias (DP \pm 21 ) dias. Nos pacientes do sexo masculino a média de tempo de desenvolvimento das lesões foi de 19 dias (DP \pm 22 ) dias e nos de sexo feminino foi de 16 (DP \pm 17 ) dias.

O teste de Friedman apontou que não houve associação entre idade e tempo de internação ( $p=0,1167)$ e que não houve associação significativa entre idade e tempo de desenvolvimento de lesão $(p=0,7066)$.

A média de lesões por paciente foi de 1,19, com variação entre 1 e 5 lesões.

Conforme os últimos dados do DATASUS (2011) a média de tempo de internação dos pacientes em hospitais de média e alta complexidade é de 9,3 dias. ${ }^{13}$ Um estudo em um hospital universitário demonstrou 20,9 dias de internação, que é menos da metade comparado ao presente estudo. ${ }^{14}$

O tempo de internação prolongado é um fator que predispõe ao surgimento de LP. Estudos apontam que esse tipo de lesão pode ocorrer em menos de três dias de internação. ${ }^{6}$ Em um estudo realizado com 5.905 pacientes de um hospital da cidade de Manaus, mostrou forte relação entre tempo de internação e estágios mais graves das lesões por pressão. ${ }^{10^{3}}$ 
Outro trabalho desenvolvido em três hospitais referências para traumatologia evidenciou que houve relevância entre tempo de internação e ocorrência de LP, sendo o maior tempo de internação um fator preditor nos pacientes de tal clínica em função das características destes, corroborando também com o presente estudo. ${ }^{15}$

As notificações apontaram que $77(37,4 \%)$ pacientes tinham uma comorbidade, $53(25,7 \%)$ duas comorbidades, $17(8,3 \%)$ três comorbidades, $5(2,4 \%)$ quatro comorbidades e $2(1 \%)$ cinco comorbidades.

Com relação às comorbidades, Palagi e colaboradores afirmam que pessoas com sensibilidade diminuída, imobilidade prolongada ou acamadas, com idade avançada são as mais susceptíveis à LP, que ocorrem mais frequentemente nas regiões mentoniana, occipital, escapular, cotovelo, sacral, ísquio, trocânter, crista ilíaca, joelho, maléolo e calcâneo. Outros fatores que aumentam o risco de desenvolver LP englobam doenças degenerativas, tolerância tecidual reduzida (pele frágil), incontinência urinária ou intestinal, e desnutrição ou obesidade. Nestes casos, é crucial a implementação de cuidados adequados, o que instiga a equipe multiprofissional a buscar capacitação para o reconhecimento do problema e para novas intervenções. ${ }^{16}$

Para correlação entre idade e o tempo de internação foi aplicado o teste de Friedman qual resultou em um coeficiente de correlação de -0,0256 e um p de 0,7066, portanto, para esses dados, não há uma associação significativa.

Os principais fatores de risco que predispõem ao desenvolvimento da LP são classificados em intrínsecos e extrínsecos. Os fatores intrínsecos normalmente são representados por idade, estado nutricional, perfusão tecidual, hidratação da pele, condições de mobilidade, nível de consciência e comorbidades associadas. Já os fatores extrínsecos estão relacionados à exposição física do paciente às condições externas, como fricção, cisalhamento, umidade e pressão, sendo essa última, o fator principal ligado ao desenvolvimento da lesão. ${ }^{11}$

A maior parte dos pacientes relacionados nas fichas de notificação (Tabela 2) eram pacientes vítimas de trauma (27,30\%), com patologias neurológicas $(26,00 \%)$ e pulmonares $(23,80 \%)$.

Tabela 2. Descrição da amostra de acordo com a clínica de internação, relacionada aos diagnósticos de base.

\begin{tabular}{|l|r|r|}
\hline Clínicas/ diagnósticos & $\mathbf{n}$ & $\%$ \\
\hline Traumatologia & 62 & 27,30 \\
\hline Neurologia/neurocirurgia & 59 & 26,00 \\
\hline Pneumologia & 54 & 23,80 \\
\hline
\end{tabular}




\begin{tabular}{|l|r|r|}
\hline Cardiovascular & 18 & 8,00 \\
\hline Cirurgia geral & 12 & 5,30 \\
\hline RNC & 5 & 2,20 \\
\hline Ortopedia & 3 & 1,80 \\
\hline Neonatologia & 3 & 1,30 \\
\hline Urologia & 1 & 0,40 \\
\hline Dermatologia & 1 & 0,40 \\
\hline Gastroenterologia/cirurgia do aparelho digestivo & 5 & 2,20 \\
\hline Outros & $\mathbf{2 2 7}$ & $\mathbf{1 0 0 , 0 0}$ \\
\hline Total & & \\
\hline
\end{tabular}

Um estudo realizado em quatros hospitais públicos de São Luís, MA, corroborou com o presente, pois demonstrou que a disfunção neurológica foi predominante nos pacientes que desenvolveram LP. As clínicas médica, cirúrgica, ortopédica e terapia intensiva são tidas como significativamente incidentes na ocorrência destas. ${ }^{17}$

Quando observado o índice de desenvolvimento de lesão por pressão, os pacientes com lesões ou patologias neurológicas, representaram $26 \%$ das fichas recebidas. Isso se dá devido à diminuição da sensibilidade e mobilidade comprometidas desses pacientes. Geralmente, pacientes neurológicos apresentam restrição de mobilidade devido a complicações, o que pode determinar aumento de pressão tecidual em determinadas regiões corporais e desenvolvendo as lesões por pressão. ${ }^{15}$

Pacientes com patologias pulmonares, como doença pulmonar obstrutiva crônica, síndrome respiratória aguda grave, pneumonia e demais doenças que afetam o sistema respiratório, geralmente necessitam de cuidados especiais, como é o caso de pacientes que necessitam permanecer em posição de prona, que apesar de fornecer um melhor fluxo pulmonar de oxigênio, faz com que os pacientes tenham aumento na pressão tecidual em áreas de proeminências ósseas, como joelhos, ombros, região frontal, região púbica e de pés. ${ }^{18}$

A distribuição das regiões corporais afetadas pelo desenvolvimento de lesões na amostra estudada está apresentada na Tabela 3, corroborando com estudos que evidenciam a topografia sacral como a mais acometida, apresentando em média $70 \%$ dos pacientes acometidos por lesão. ${ }^{3}$ 
Tabela 3. Distribuição das lesões notificadas de acordo com a região corporal de localização.

\begin{tabular}{|l|r|r|}
\hline Localização & $\mathbf{n}$ & \% \\
\hline Sacro & 107 & 47,14 \\
\hline Calcâneo & 20 & 8,81 \\
\hline Occipital & 19 & 8,37 \\
\hline Trocânter & 14 & 6,17 \\
\hline Glúteo & 9 & 3,96 \\
\hline Escápula & 8 & 3,52 \\
\hline Orelha & 6 & 2,64 \\
\hline Tórax & 6 & 2,64 \\
\hline Face & 5 & 2,20 \\
\hline Narina & 5 & 2,20 \\
\hline Lábio & 4 & 1,76 \\
\hline Cotovelo & 4 & 1,76 \\
\hline Pênis & 3 & 1,32 \\
\hline Língua & 2 & 0,88 \\
\hline Joelho & 1 & 0,44 \\
\hline Maléolo & 2 & 0,88 \\
\hline Mento & 2 & 0,88 \\
\hline Cervical & 1 & 0,88 \\
\hline Pálpebra & 1 & 0,88 \\
\hline Coluna Torácica & 2 & 0,44 \\
\hline Supra Púbica & 1 & 0,44 \\
\hline Ombro & & 0,44 \\
\hline Panturrilha & & 0,44 \\
\hline Esterno & & \\
\hline Local não relatado & & \\
\hline
\end{tabular}




\begin{tabular}{|l|r|r|}
\hline Total & 227 & 100,00 \\
\hline
\end{tabular}

Com relação à classificação das lesões (Tabela 4), destaca-se que a maior parte delas não estavam classificadas (43,17\%).

Tabela 4. Distribuição das lesões notificadas, de acordo com a classificação, 2019.

\begin{tabular}{|l|r|r|}
\hline Classificação da lesão & $\mathbf{n}$ & \% \\
\hline Estágio 1 & 74 & 32,60 \\
\hline Estágio 2 & 42 & 18,50 \\
\hline Estágio 3 & 6 & 2,64 \\
\hline Escara & 7 & 3,08 \\
\hline Classificação não relatada & 98 & 43,17 \\
\hline Total & $\mathbf{2 2 7}$ & $\mathbf{1 0 0 , 0 0}$ \\
\hline
\end{tabular}

Lesões em estágio 1 perfizeram 32,60\% dos pacientes, e em estágio 2 18,50\%, dados quais comparados com variável analisada em outra pesquisa, que englobou pacientes internados em diversos hospitais, evidenciou que os estágios mais frequentes foram estágio 1 e $2 .^{7}$

As subnotificações influenciam diretamente na prática clínica, uma vez que a notificações de eventos adversos norteiam o desenvolvimento de práticas com qualidade. Sugere-se que a gestão de processos de trabalho seja melhorada pautada por um sistema organizacional que fomente o desenvolvimento de notificações como instrumento de auxílio, baseado sempre em evidências práticas de segurança do paciente. Essas melhorias devem ser baseadas em abordagem sistemática e aprendizagem a partir de erros no sentido de capacitar os profissionais frente a ocorrência de eventos adversos influenciando diretamente na redução dos danos. ${ }^{19}$

O desfecho do paciente foi outro dado destacado neste estudo, sendo que 138 (61\%) pacientes obtiveram alta e 89 (39\%) pacientes evoluíram a óbito. O teste qui-quadrado resultou em uma estatística de 1,9150 e p-valor=0,2116, portanto não houve uma associação significativa entre idade e desfecho, os resultados são independentes entre si. Estudos apontam que em contrapartida, a incidência de óbitos nos pacientes que desenvolveram lesões por pressão entre $53,85 \%$ a $75,00 \%$ mostrando que as lesões por pressão podem desencadear complicações graves, que resultam em maior sofrimento físico e 
emocional, aumento de tempo de internação e aumento da taxa de mortalidade. ${ }^{20,21}$

Com relação ao sexo do paciente e ao desfecho, o teste qui-quadrado com correção de continuidade resultou em uma estatística de 1,9150, portanto, sexo e desfecho são resultados independentes entre si. Foi possível observar nos demais estudos sobre a temática, que a maioria dos indivíduos com LP eram do sexo masculino, esse fato também foi encontrado em um Hospital de Portugal. Igualmente, outras pesquisas observaram tal informação, porém, assim como referido trabalho, não houveram indícios estatisticamente significativos que relacionem a variável sexo com a gênese de LP, o que sugere que os dados encontrados estejam mais correlacionados ao tipo de especificidade do hospital, o qual é referência em atendimentos ortopédicos e traumatologia, saúde do trabalhador, especialidades da saúde fortemente associadas à acidentes de trânsito, nos quais a maioria dos pacientes são indivíduos jovens e do sexo masculino. ${ }^{10}$

\section{CONCLUSÃO}

As fichas de notificação de lesões mostraram que a maior parte dos pacientes eram do sexo masculino, com idade média de 54,2 anos, brancos e ficaram internados em média 45 dias. Comorbidades estavam presentes em $74,8 \%$ dos pacientes, o que pode ser um fator de risco para a lesão por pressão. A grande maioria dos pacientes eram pacientes das clínicas de traumatologia e neurologia.

A média de lesão por paciente foi de 1,9 e a localização mais frequente foi a sacral.

Houve subnotificação com relação ao estadiamento das lesões, o que pode gerar questionamentos sobre a razão do ocorrido e evidenciar uma lacuna para a prática assistencial e para o controle de complicações do cuidado em saúde.

O conhecimento da realidade institucional proporciona ao profissional base cientifica para tomada de decisões.

A avaliação de risco de desenvolvimento de LP é uma ferramenta eficaz na prevenção deste tipo de complicação da hospitalização. A avaliação do risco é um instrumento que pode nortear as ações da equipe de saúde, visando a identificação de ações preventivas. Com isso, trabalha-se a partir da avaliação epidemiológica do surgimento das lesões, sendo assim, a notificação é uma ferramenta primordial para prevenção, visto que o delineamento destas lesões faz com que haja maior entendimento sobre o público ao qual norteia a prática clínica na unidade na instituição, contribuindo para promoção e prevenção das LP. 


\section{REFERÊNCIAS}

1. National Pressure Ulcer Advisory Panel. National Pressure Ulcer Advisory Panel (NPUAP) announces a change in terminology from pressure ulcer to pressure injury and updates the stages of pressure injury, 2016. Disponível em: https://www.npuap.org/national-pressure-ulcer-advisory-panel-npuapannounces-a-change-in-terminology-from-pressure-ulcer-to-pressure-injuryand-updates-the-stages-of-pressure-injury/

2. Mendonça, PK et al. Prevenção de Lesão por Pressão: ações prescritas por enfermeiros de centros de terapia intensiva. Texto \& Contexto Enfermagem, 2018. 27(4): 2-10. DOI: http://dx.doi.org/10.1590/0104-07072018004610017.

3. Moore, ZE; Cowman, S. Repositioning for treating pressure ulcers. Cochrane Database of Systematic Reviews, 2015. Disponível em: https://www.cochranelibrary.com/cdsr/doi/10.1002/14651858.CD006898.pub 4/epdf/full

4. Palagi, S; Severo, IM, Menegon DB; Lucena AF. Laser therapy in pressure ulcers: evaluation by the Pressure Ulcer Scale for Healing and Nursing Outcomes Classification. Rev Esc Enferm USP, 2015. 49(5):826-833. DOI: http://dx.doi.org/10.1590/S0080-623420150000500017

5. Petz, FFC,et al. Úlcera por pressão em unidade de terapia intensiva: estudo epidemiológico. Revista de Enfermagem, 2017. 11(1); 287-293. DOI: 10.5205/reuol.7995-69931-4-SM.1101sup201706.

6. Otto, $\mathrm{C}$ et. al. Fatores de Risco para o desenvolvimento de Lesão Por Pressão em pacientes críticos. Enfermagem em Foco, 2018. 10(1); 07-11. Disponível em: http://revista.cofen.gov.br/index.php/enfermagem/article/view/1323

7. Jiang, Qet al. The incidence, risk factors and characteristics of pressure ulcers in hospitalized patients in China. International journal of clinical and experimental pathology, 2017. 7(5):2587-2594. ISSN:19362625/IJCEP1402017

8. National Pressure Ulcer Advisory Panel. Pressure Ulcer Stages Revised. Washington, 2016. Disponível em: https://www.npuap.org

9. Moraes JT et. al. Conceito e classificação de lesão por pressão: atualização do National Pressure Ulcer Advisory Panel. RECOM. 2016.6(2); 2292-306. DOI: http://dx.doi.org/10.19175/recom.v6i2.1423

10. Mendonça ASGB, Rocha ACS, Fernandes TG. Perfil epidemiológico e clínico de pacientes internados com lesão por pressão em hospital de referência no Amazonas. Revista de Epidemiologia e Controle de Infecção, 2018. 8(3); 253-260. DOI: http://dx.doi.org/10.17058/reci.v8i3.11857

11. Galvão NS, Lopes ND, Oliveira APP. Aspectos epidemiológicos e clínicos de pacientes com úlcera por pressão internados em uma instituição hospitalar. Rev. da Associação Brasileira de Estomaterapia, Ostomias, 
Feridas e Incontinências, 2015. Disponível em:

https://www.revistaestima.com.br/index.php/estima/article/view/106

12. Araújo TM, Araújo MFM, Caetano JA. Comparação de escalas de avaliação de risco para úlcera por pressão em pacientes em estado crítico. Acta Paulista de Enfermagem, 2011. 24(5):695-700. DOI:

http://dx.doi.org/10.1590/s0103-21002011000500016

13.DATASUS. Brasil, 2011. Sistema de informações hospitalares. Disponível em: www.datasus.gov.br.

14. Rufino GP et al. Avaliação de fatores determinantes do tempo de internação em clínica médica. Ver Bras Clin Med, 2012. Disponível em: http://www.sbcm.org.br/revistas/RBCM/RBCM-2012-04.pdf\#page=26

15. Araujo GL, Whitaker IY. Morbidade hospitalar de motociclistas acidentados: fatores associados ao tempo de internação. Acta Paulista de Enfermagem, 2016. 9(2):178-84. DOI: http://dx.doi.org/10.1590/1982-0194201600025

16. Palagi S, Severo IM, Menegon DB, Lucena AF. Laser therapy in pressure ulcers: evaluation by the Pressure Ulcer Scale for Healing and Nursing Outcomes Classification. Rev Esc Enferm USP, 2015; 49(5):826-833. DOI: 10.1590/S0080-623420150000500017.

17. Santos ACM. Perfil epidemiológico de pacientes com lesão por pressão estágios III e IV. 2018. 62 f. TCC (Graduação) - Curso de Enfermagem, Enfermagem, Universidade Federal do Maranhão, 2018. Disponível em: https://monografias.ufma.br/jspui/handle/123456789/2053

18. Girard $\mathrm{R}$ et al. The impact of patient positioning on pressure ulcers in patients with severe ARDS: results from a multi centre randomised controlled trial on prone positioning. Intensive Care Medicine. 40:397-403. DOI: http://dx.doi.org/10.1007/s00134-013-3188-1

19. Moreira IA et al. Conhecimento dos profissionais de saúde sobre eventos adversos em unidade de terapia intensiva. Revista Enfermagem. 23(4):461 7. DOI: http://dx.doi.org/10.12957/reuerj.2015.5158

20. Candaten AE, Vieira YB, Barcellos RA. Incidência de Lesões Por Pressão em Pacientes Internados em Unidades de Terapia Intensiva. Rev. UNINGÁ, 2019. Disponível em:

http://revista.uninga.br/index.php/uninga/article/view/1455/1899.

21. Silva MLN. et al. Úlcera por pressão em unidade de terapia intensiva: análise da incidência e lesões instaladas. Rev Rene, 2013; 14(5):938-44. Disponível em: http://www.periodicos.ufc.br/rene/article/view/3623/2865 
Recebido: 24 de setembro de 2019. Aceito: 22 de novembro de 2019

Correspondência: Josni Tauffer. E-mail: josnitauffer@hotmail.com

Conflito de Interesses: os autores declararam não haver conflito de interesses.

(C) This is an Open Access article distributed under the terms of the Creative Commons Attribution License, which permits unrestricted use, distribution, and reproduction in any medium, provided the original work is properly cited 